\title{
Sforzesco brace (SPoRT Concept) versus Risser cast in adolescent idiopathic scoliosis treatment: similar efficacy, with reduced spinal side effects for the brace
}

\author{
Stefano Negrini* ${ }^{*}$, Fabio Zaina1 ${ }^{1}$, Francesco Negrini ${ }^{1}$, Gianfranco Marchini ${ }^{2}$ \\ and Angelo Aulisa ${ }^{3}$
}

Address: ${ }^{1}$ ISICO (Italian Scientific Spine Institute), Via Carlo Crivelli 20, 20122 Milan, Italy, ${ }^{2}$ COL, Milan, Italy and ${ }^{3}$ Rome, Italy

Email: Stefano Negrini* - stefano.negrini@isico.it

* Corresponding author

from 4th International Conference on Conservative Management of Spinal Deformities

Boston, MA, USA. 13-16 May 2007

Published: 12 October 2007

Scoliosis 2007, 2(SuppI I):S20 doi:I0.II86/I748-7I6I-2-SI-S20

This abstract is available from: http://www.scoliosisjournal.com/content/2/SI/S20

(c) 2007 Negrini et al; licensee BioMed Central Ltd.

\section{Objective}

To compare the results of the recently introduced Sforzesco brace with the classical Risser cast in the treatment of adolescent idiopathic scoliosis (AIS).

\section{Study design}

From our prospective database we included all patients with AIS, followed-up for eighteen months during Risser cast (twelve months plus six month in Lyon brace full time) or Sforzesco brace treatments. Risser cast treatment [1] was our standard treatment for worst curves until January 2004. The Sforzesco brace method is our current approach. We had eighteen patients in cast (83\% female, $14.3 \pm 1.11$ years of age, Cobb angle $39.5 \pm 7.5$ degrees) and thirty-three patients in brace (79\% females, $14.1 \pm$ 1.10 years of age, Cobb angle $40.9 \pm 12.1)$. Analysis included measurement of Cobb angle, Bunnell angle of trunk rotation (ATR) [2], rib hump magnitude, aesthetic index, and sagittal distances from the plumbline.

\section{Results}

No significant differences were present between the two groups, at the start. After treatment, mean values for clinical parameters between the two groups were statistically distinct. An exception was thoracic Cobb angle (+1 degree) for cast treated patients, and thoraco-lumbar Cobb angle $(-5.4$ degrees, $\mathrm{p}=\mathrm{NS})$ for brace treated patients. Comparing the groups, Cobb reduction was higher for brace ( -5 degrees vs -3.5 degrees) but the difference was statistically significant only for thoracic curves. The cast achieved better results on ATR and rib hump, but resulted in a significant sagittal curve reduction $(-11 \mathrm{~mm}$ at C7 $\mathrm{p}<0.001$, versus $-3 \mathrm{~mm} \mathrm{p}=\mathrm{NS}$ ).

\section{Conclusion}

Clinical results with the Sforzesco brace are similar to results with the Risser cast. Considering the reduced costs, both personal (Quality of Life) and social (outpatient only for the brace, versus four weeks' inpatient treatment for the Risser cast), brace treatment should be preferred.

\section{References}

I. Lonstein JE: Cast techniques. In Moe's Textbook of Scoliosis and Other Spinal Deformities Volume Chapter 9. Edited by: Lonstein JE, Bradford DS, Winter RB, Ogilvie JW. WB. Saunders Company : I I 7-I32.

2. Bunnell WP: An objective criterion for scoliosis screening. J Bone Joint Surg 1984, 66A: I38I-I 387. 